\title{
Evaluation of Quantitative Liver Function Tests in HIV-Positive Patients under Anti-Retroviral Therapy
}

\author{
M. Miller ${ }^{1}$, A. Kahraman ${ }^{1,2}$, B. Ross ${ }^{1,3}$, M. Beste ${ }^{1}$, G. Gerken $^{1}$ \\ ${ }^{1}$ Department of Gastroenterology and Hepatology, University Hospital Essen, Germany, \\ ${ }^{2}$ Miles and Shirley Fitterman Center for Digestive Diseases, Division of Gastroenterology and Hepatology, Mayo Clinic College of \\ Medicine, Rochester, Minnesota, USA, \\ ${ }^{3}$ STD-Unit, Department of Dermatology and Venerology, University Hospital Essen, Germany
}

\begin{abstract}
Background and Aims: Quantitative tests of liver function (QTLF) which are based on the hepatic metabolism or clearance of test substances have been successfully used to predict prognosis of a variety of different liver diseases. Still sufficient data in HIV-patients under anti-retroviral therapy (ART) are lacking. Therefore, the aim of this prospective study was to investigate if and to what extent ART influences a broad panel of quantitative tests of liver function in patients with HIV-infection.
\end{abstract}

Patients and Methods: Nineteen patients (14 males, 5 females, mean age 40 years) with HIV-infection under went QTLF including lidocaine half-life test (LHT), galactose elimination capacity (GEC), and indocyanine green clearance (IGC). These tests were performed before and 3 to 6 months after initiation of anti-retroviral therapy. Twenty age-matched healthy, medicationand virus-free adults served as controls.

Results: Lidocaine half-life was significantly lower in HIV-patients without ART. Combining anti-retroviral therapies shifted cytochrome p450 activity back into standard ranges. Galactose elimination capacity as a parameter of cytosolic liver function and indocyanine green clearance as a parameter of liver perfusion were not affected by ART.

Conclusions: QTLF may be a tool to predict prognosis or hepatic complications in HIV-infected patients with liver disease. Early determination of lidocaine half-life seems to be useful - this should be considered during the treatment of HIV-positive individuals.

Key words: anti-retroviral therapy, HIV, quantitative tests of liver function

Abbreviations: ART, anti-retroviral therapy; GEC, Galactose elimination capacity; HAART, highly active anti-retroviral therapy; $\mathrm{HCV}$, hepatitis $\mathrm{C}$ virus; HIV, human immunodeficiency virus; IGC, Indocyanine green clearance; LHT, Lidocaine half-life test; QTLF, quantitative tests of liver function

\section{INTRODUCTION}

Liver toxicity is one of the most relevant adverse ef fect of anti-retroviral therapy (ART) in patients infected with human immunodeficiency virus type-1 (HIV-1) (Lebovics, Thung et al. 1985; Schneiderman, Arenson et al. 1987). Although there are many other causes of liver disease in these patients (Farrell 2002), the most common are viral co-infections, drugs and narcotics, which are most prominent upon treatment with highly active anti-retroviral therapy (HAART). Likewise, both nucleoside reverse transcriptase inhibitors (NRTI) and protease inhibitors (PI) are associated with metabolic alterations and disorders, such as liver toxicity, lipodystrophy and insulin resistance (Ena, Amador et al. 2003; Lee, Seneviratne et al. 2004). The spectrum of liver damage in this population ranges from simple hepatic steatosis to steatohepatitis and even fibrosis (Clark, Brancati et al. 2002). Most patients with hepatic alterations are asymptomatic, and liver disease may unexpectedly be diagnosed during the evaluation of unexplained persistent elevations of serum liver enzymes In this case, and once other known aetiologies have been ruled out, imaging procedures often suggest fatty infiltration of the liver parenchyma (Yano, Tagawa et al. 2001). Since most of these patients are indolent, fatty liver seems to be a benign and stable disease (Reid 2001). Nevertheless, sensitizing the liver may lead to acute-on-chronic liver failure (Kahraman, Miller et al. 2006) or cryptogenic cirrhosis with the necessity of liver transplantation. Consequently, it is of major clinical importance to assess stage and prognosis of liver disease in HIV-positive patients.

Conventional serum parameters (e. g., coagulation factors, albumin or cholinesterase) often lack sensitivity in early stages of liver disease and are mostly changed in advanced stages. However, the available routine laboratory tests only reflect individual facets of liver in jury, synthetic capability or a pre-existing disease. They are not able to identify patients with marginal liver function. Various laboratory data and imaging techniques have been used to complement the Child-Turcotte-Pugh or the MELD score to predict severity of liver disease and assess functional hepatic reserve. Therefore, quantitative tests of liver function (QTLF) may be a useful diagnostic tool to predict prognosis and outcome of liver disease in HIV-infected patients under anti-retroviral therapy, and they may be useful to search for drugs that restore liver function.

Quantitative or dynamic liver function tests were in troduced in the middle of the last century (Bradley, In-

*Michael Miller and Alisan Kahraman contributed equally to this study. 
gelfinger et al. 1945; Leevy, Mendenhall et al. 1962 Tygstrup 1963). They measure the turn-over or elimination of an exogenous test substance that is exclusively metabolized and eliminated by the liver, and have helped to elucidate the structure-function relationship of the liver. Furthermore, they have been shown to have a prognostic value independent of and often superior to that of scores like the Child-Turcotte-Pugh or the recently used MELD score (Reichen 1995). All quantitative tests are based on the hepatic clearance principle. The clearance of the test substance is determined by the liver blood flow via the portal vein and the hepatic artery, as well as by the degree to which this test substance is extracted by the liver, whereas the final elimination depends on the hepatic metabolic capacity. Depending on the administered compound, it is possible to separately analyze microsomal (e. g., aminopyrine, caffeine, erythromycin, methacetin, phenacetin) cytosolic (galactose, phenylalanine) or mitochondria $(\alpha$-ketoisocaproic acid, methionine) metabolic pathways. Most available data come from microsomal function tests; data about cytosolic and mitochondrial liver function are more limited. The effects of liver perfusion-dependent metabolism, induction or inhibition of cytochrome p450, nutrition or age provide little dependable information to evaluate functional liver mass (Armuzzi, Candelli et al. 2002; Schneider, Caspary et al. 2004)

QTLF include for example the lidocaine half-life test (LHT), the galactose elimination capacity (GEC), and the indocyanine green clearance (IGC). LHT reflects the function of the cytochrome 450 system and the quality of detoxification of liver cells (Lamesch Ringe et al. 1990; Reichel, Wienkoop et al. 1995; Reichel, Wienkoop et al. 1995; Jochum, Beste et al. 2006). Disappearance of lidocaine is dependent upon liver perfusion as well as metabolism by different cytochrome p450 isoenzymes. GEC reveals the metabolic liver function (Tygstrup 1966; Tygstrup, Andersen et al. 1985; Keiding 1987) whereas IGC provides informa tion about the hepatic perfusion and the excretion out put of the bile, at least in healthy livers (Leevy, Mendenhall et al. 1962; Meijer, Weert et al. 1988; Plevris, Jalan et al. 1999; Ott, Clemmesen et al. 2000)

So far, data about QTLF are existent for patients with liver cirrhosis due to chronic $\mathrm{HCV}$-infection to as- sess disease severity, for patients with liver cirrhosis to discriminate between different aetiologies, for patients with chronic hepatitis $\mathrm{B}$ and $\mathrm{C}$ co-infection in relation to fibrosis, for patients with non-cirrhotic chronic hepatitis $\mathrm{C}$-infection to predict portal vein velocity, and finally for living liver donors and their recipients to assess functional hepatic reserve (Herold, Berg et al 2000; Herold, Heinz et al. 2001; Herold, Heinz et al. 2001; Herold, Ganslmaver et al. 2002; Herold, Regn et al. 2002; Herold, Ganslmayer et al. 2003; Jochum, Beste et al. 2006; Reichen 2006). However, still sufficient data in HIV-patients comparing several quantitative tests of liver function are lacking, and to date no clinical study has been carried out to assess the values of these tests in patients with HIV-infection. We there fore tested the hypothesis if liver function might be restored in patients with HIV-infection following antiretroviral therapy. Here, we investigated if and to what extent changes in either hepatic metabolic enzyme function and in liver perfusion can be achieved by antiretroviral therapy, and also if changes in QTLF may help to discriminate responders from non-responders to anti-viral treatment strategies in patients with HIV infection. We applied the panel of LHT, GEC and IGC to 19 patients with HIV-infection under therapy with different anti-viral regimes and compared these results to a healthy, medication- and virus-free control group Beside the evaluation of the liver function under anti retroviral therapy, we especially paid attention to the liver function of HIV-patients with HCV co-infection under ART since infection with the more liver-specific $H C V$ can compromise liver function.

\section{Patients and Methods}

Patients: Nineteen consecutive patients of all risk classes $(14$ males and 5 females, mean age $40 \pm 11$. years) with HIV-infection were included in this prospective study (Table 1). Among these HIV-patients, 6 were classified C3, 9 were classified B1/2, and finally, 4 were classified $A 1 / 2$ according to the CDCclassification of 1993. Values for CD4+ T cells were between 70 to $1200 / \mu$ and viral load ranged between $<50$ to 360.000 copies $/ \mathrm{ml}$. Moreover, 7 of these patients had a HCV co-infection. Three patients were trea-

Table 1. Demographic and clinical characteristics of patients and controls investigated in the study.

\begin{tabular}{ccc}
\hline & $\begin{array}{c}\text { Healthy controls } \\
(\mathrm{n}=20)\end{array}$ & $\begin{array}{c}\text { Patients with HIV } \\
(\mathrm{n}=19)\end{array}$ \\
\hline Gender ratio & $\mathrm{m}: \mathrm{f}=15: 5$ & $\mathrm{~m}: \mathrm{f}=14: 5$ \\
Age (yrs.) & $42.1 \pm 7.5$ & $40 \pm 11.3$ \\
Weight $(\mathrm{kg})$ & $69.7 \pm 16.1$ & $64 \pm 17.4$ \\
Height $(\mathrm{cm})$ & $171.4 \pm 11.5$ & $174.7 \pm 11.5$ \\
BMI $\left(\mathrm{kg} / \mathrm{m}^{2}\right)$ & $22.5 \pm 1.3$ & $19.6 \pm 4.6$ \\
AST $(\mathrm{mg} / \mathrm{dl})$ & $24.5 \pm 1.7$ & $29 \pm 11.3$ \\
ALT $(\mathrm{mg} / \mathrm{dl})$ & $28.7 \pm 2.8$ & $27.3 \pm 17.2$ \\
Total bilirubin $(\mathrm{mg} / \mathrm{dl})$ & $0.5 \pm 0.3$ & $0.5 \pm 0.2$ \\
$\gamma-G T$ & $52.1 \pm 5.2$ & $97.8 \pm 11.5$ \\
HCV co-infection & none & 7 \\
\hline
\end{tabular}


ted with NRTI (nucleoside reverse transcription inhibitor) alone, 4 with NRTI + PI (protease inhibitor), 4 with NRTI + NNRTI (non-NRTI), 5 with NRTI + NNRT + PI, and finally, 3 patients were without ART. Chronic alcohol abuse was not observed in any of these patients ( $<60 \mathrm{~g} /$ day in men and $<30 \mathrm{~g} /$ day in women). Patients other than Caucasian origin, age $<18$ or $>65$ years, active drug abuse, and other causes of liver disease were excluded. In addition to exploration of the case history and the clinical status, chemical laboratory values were determined for all HIV-patients. Twenty age- and gen der-matched bealthy, medication- and virus-free adults served as controls 15 males and 5 females, mean age $42.1 \pm 7.5$ years), and all values of QTLF in HIV patients were correlated with the normal values of this group (Table 1). To assess the condition of the liver in the control group, several liver parameters including serum transaminases, total bilirubin, $\gamma-G T$, serum bile acids, albumin, and routine coagulation tests were determined, which were within normal ranges.

All patients underwent QTLF before and 3 to 6 months after the initiation of anti-retroviral therapy.

The current study was performed in accordance to the ethical standards formulated in the version of the 1964 Declaration of Helsinki. All patients gave their informed consent prior to inclusion in this present study. Before performance of the QTLF, all drugs (e. g., barbiturates, benzodiazepines, anti-depressive drugs, H2-blockers, grapefruit juice, etc.) that could potentially interfere with liver function were discontinued in both groups 4 weeks in advance.

Measurement and calculation of LHT, GEC and

IGC: The following three pharmacokinetic tests were performed to quantitatively assess changes in liver function during anti-retroviral therapy for each patient. Briefly, lidocaine (xylocaine $2 \%$, Firma Astra Wedel, Germany) was given as a single bolus injection of $1 \mathrm{mg} / \mathrm{kg}$ body weight over 2 minutes, $5 \mathrm{ml}$ blood samples were obtained from a peripheral vein at 30, 60, 120, 180, 240, and finally $300 \mathrm{~min}$., and detected in the serum using the Fluorescence Polarization Immunoassay Method by the Abbott TDX-System (Firma Abbott, Wiesbaden, Germany). Lidocaine concen trations were expressed in $\mu \mathrm{g} / \mathrm{ml}$ and were calculated against an internal calibration curve. Background concentrations measured before infusion of lidocaine were subtracted from all results. The assay used had a limit of detection of $5 \mathrm{ng} / \mathrm{ml}$. Lidocaine half-life was expressed in minutes.

To determine GEC, galactose (Pharmaceutical Institute, University of Essen, Germany) was injected intravenously as a bolus (45\% galactose, $0.5 \mathrm{~g} / \mathrm{kg}$ bod weight), which saturates the catabolic enzyme system. The speed at which elimination of galactose takes place is determined by its phosphorylation by galactokinase which is located in the hepatic cytoplasm. Serum sam ples were treated with $1 \mathrm{ml}$ of $0.33 \mathrm{M}$ perchloric acic per $200 \mu \mathrm{l}$ serum and centrifuged at $2.400 \mathrm{~g}$. The supernatant was analyzed using the Enzymatic Test Ki Galactose (Firma Roche Diagnostics GmbH, Mannheim, Germany). Urine was collected over a period of 5 hours after injection of galactose. The volume of the collected urine was measured, and for calculation of GEC at time point 0 and from 20 to 60 minutes (5- minutes intervals), serum concentrations of galactose were determined photospectrometrically (PM6, Firma Zeiss, Oberkochem, Germany) at $366 \mathrm{~nm}$. GEC wa calculated using the following formula: (infusion dose [g] galactose - galactose in urine $[\mathrm{g}]$ )/(time at concentration $0+$ infusion time) $\times 1.000 / \mathrm{kg}$ body weight. The results were expressed as $\mathrm{mg} / \mathrm{kg} /$ minute.

IGC is absorbed exclusively by the liver and, if there is a high hepatic elimination rate, the substrate is quickly excreted in a mostly unmodified form by $97 \%$ in the bile. Therefore, IGC is influenced both by the biliary secretion capacity and by the liver perfusion. In addition, IGC is considered to be insensitive to pharmacological influences because its metabolism solely depends on the hepatic transport and biliary secretion. This test is the most widely used clearance test for liver diseases. Nevertheless, it remains imperfect because it depends both on the hepatic blood flow and on the functional capacity of the liver. IGC (Cardio Green, Firma Paesel, Hamburg, Germany) is also injected in travenously as a bolus of $0.5 \mathrm{mg} / \mathrm{kg}$ body weight. Serum concentrations were also measured photospec trometrically at $800 \mathrm{~nm}$ every 3 minutes until $21 \mathrm{~min}$ utes post-injection. Values were expressed as half-life in minutes. Serum transaminase levels, parameters of cholestasis, and coagulation factors were determined by routine methods at the Department of Medical Chemistry, University of Essen.

Statistical Analysis: All data are expressed as the mean \pm standard error unless otherwise indicated. For statistical analyses, the Mann-Whitney U test for unrelated groups was used to compare HIV-patients and healthy controls at different time points. For compari son within these 2 groups at different time points, we used the Wilcoxon test for connected samples. Differences between groups were compared using analysis of variance (ANOVA) for repeated measures and post-hoc Bonferroni test to correct for multiple comparisons. Calculations were made using Microsoft Excel software and publicly available internet sources (In-Stat Soft ware, Graph Pad, San Diego, California). A p value less than 0.05 was considered to be statistically significant compared to the controls.

\section{RESULTS}

Lidocaine half-life is significantly lower in HIVpositive patients without ART: We first investigated the influence of different anti-retroviral regimens on lidocaine half-life. As demonstrated in Figure $1 A$, in bealthy controls and in HIV-positive patients without ART lidocaine half-life was significantly lower than in each treated sub-group of patients with HIV-infection suggesting a higher activation of the cytochrome $p 450$ system. Lidocaine half-life was measured before initiation and after 6 months of treatment. HIV-positive patients treated for 6 months with anti-retroviral therap had a prolonged lidocaine half-life as compared to the decreased values in the HIV-infected naïve group. Lidocaine half-life before the initiation of $A R T$ is also depicted. This increased activation in treated patients with HIV-infection might be a virus-induced effect. In addition, combining anti-retroviral therapies (Triple-Nuc, NRTI + NNRTI or NRTI + PI) shifted cytochrome 

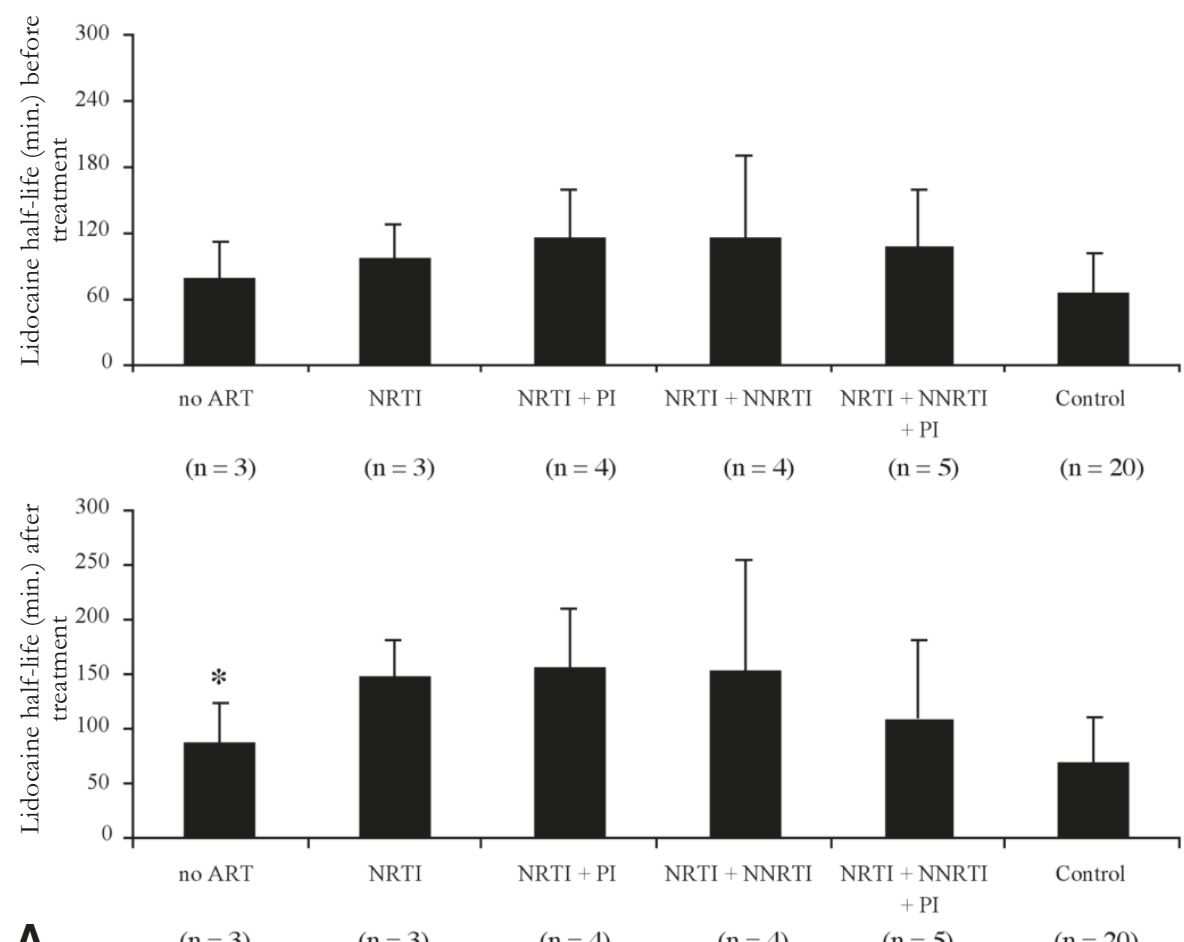

A

$(\mathrm{n}=3)$

$(\mathrm{n}=4)$

$(\mathrm{n}=4)$

$(\mathrm{n}=5)$

$(\mathrm{n}=20)$
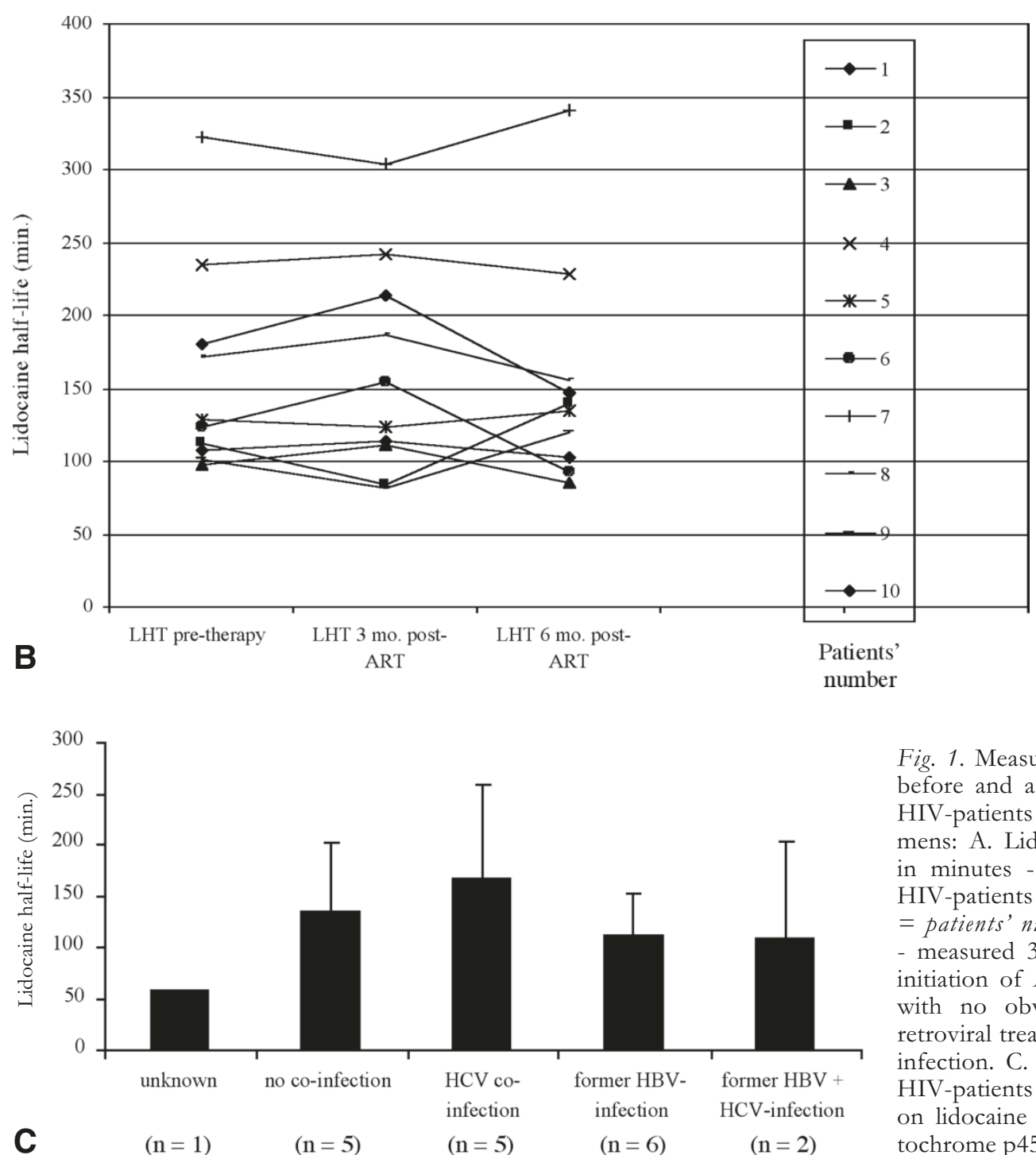

Fig. 1. Measurement of lidocaine half-life before and after initiation of therapy in HIV-patients with various anti-viral regimens: A Lidocaine half-life - expressed in minutes - was significantly lower in HIV-patients without ART * $p<0.05, n$ patients' number. B. Lidoca patients' number. B. Lidocaine half-life measured 3 and 6 months (mo.) after nitiation of ART - was almost identical with no obvious changes under antietroviral treatment in patients with HIVinfection. $C$. Hepatitis $C$ co-infection in HIV-patients had no significant influence

C $(\mathrm{n}=$

$(\mathrm{n}=5)$ $(\mathrm{n}=5)$ $(\mathrm{n}=6)$ $(\mathrm{n}=2)$ on lidocaine half-life and activity of cytochrome $\mathrm{p} 450$ 
Table 2. Markers of liver function in HIV-patients with and without HCV co-infection ( $\mathrm{n}=$ patients' number).

\begin{tabular}{cccc}
\hline & $\begin{array}{c}\text { HIV with HCV co-infection } \\
(\mathrm{n}=5)\end{array}$ & $\begin{array}{c}\text { HIV without HCV co-infection } \\
(\mathrm{n}=5)\end{array}$ & p-value \\
\hline ALT (U/l) & $62.6 \pm 21.14$ & $41.65 \pm 9.75$ & 0.39 \\
AST (U/l) & $42.7 \pm 9$ & $22.55 \pm 3.77$ & 0.021 \\
$\gamma$-GT (U/l) & $138.8 \pm 43.27$ & $61.15 \pm 17.32$ & 0.056 \\
Bilirubin (mg/dl) & $0.94 \pm 0.27$ & $0.59 \pm 0.07$ & 0.083 \\
CHE (U/l) & $5.16 \pm 0.77$ & $6.21 \pm 0.36$ & 0.25 \\
\hline
\end{tabular}

p450 activity back into standard ranges. Interestingly, no obvious differences according to the various ART combinations could be demonstrated.

Lidocaine half-life test following ART was only marginally changed during the course of six months: Next, we investigated if and to what exten lidocaine half-life changed in HIV-patients upon treat ment with ART for both 3 and 6 months. Lidocaine half-life test was performed in 10 randomly selected patients out of 19 with HIV-infection. As shown in Fig ure $1 \mathrm{~B}$, lidocaine half-life was insignificantly changed before the initiation of anti-retroviral therapy and during the follow-up examination, and decreased from 161.5 (range 82-304) to 154.7 (range 85-341) minutes $(p=0.84)$. Further, lidocaine half-life test showed no significant changes during the follow-up examination in patients with HIV-infection as compared to the healthy control (range 90-322 minutes).

The monoethylglycinexylidide (MEGX) test is a dynamic test based on the conversion of lidocaine to MEGX primarily by cytochrome p450 $3 A 4$ (CYP3 A4). Losses of hepatic CYP450 3 A4 activity or changes in hepatic blood flow result in decreased MEGX formation. Previous studies have revealed that the MEGX test is an effective tool for assessing liver function and furthermore, this test has a bigh reliability when repeated (Oellerich and Armstrong 2001). We therefore investigated the prognostic value of the MEGX test in our study population. The test was performed before and 6 months after the initiation of ART, and re sults were compared to values obtained from bealthy controls. However, we found no significant changes in HIVpatients treated with various combinations as compared to bealthy controls without medication $(49.9 \pm 25.48$ $\mu \mathrm{g} / \mathrm{l}$ versus $66.29 \pm 38.55 \mu \mathrm{g} / \mathrm{l}, \mathrm{p}=0.23$ ).

Co-infection with hepatitis $\mathrm{C}$ virus in patients with HIV-infection had no influence on lidocaine halflife and activity of cytochrome p450: Within the 19 HIV-patients in our study, 5 of them were co-infected with HCV. Six patients had a former hepatitis B-, and 2 patients had a former hepatitis B- and C co-infection (Fig. 1C). Lidocaine half-life in dependence to $\mathrm{HCV}$ co infection was only slightly increased but not significantly affected, suggesting that hepatitis $C$ infection might be negligible on the liver function of HIV-patients, and is not a contra-indication for anti-retroviral therapy, although hepatitis $C$ virus is more liver specific and can compromise quality of liver function. However, this result should be interpreted with caution because of the limited number of patients investigated. Determination of other markers of liver function such as transaminases, to- tal bilirubin, and cholinesterase (CHE) revealed significant changes only for AST values in HIV -infected patients with HCV co-infection as compared to HIV-patients without hepatitis $C$ infection (Table 2).

Indocyanine green clearance was unaffected by different anti-retroviral medications in HIV-patients: Indocyanine green clearance was performed before and 6 months after initiation of HAART to investigate the correlation between hepatic perfusion and different anti-retroviral regimens in patients with HIV-infection. Three HIV-patients were without ART, 3 further patients were either treated with NRTI or with NRTI in combination with PI, 4 patients were treated with NRTI + NNRTI, and finally, 5 patients were treated with the combination of NRTI + NNRTI + PI. $A$ depicted in Figure $2 A$ and $B$, no correlation could be found between indocyanine green clearance and various anti-retroviral regimens in HIV-patients.
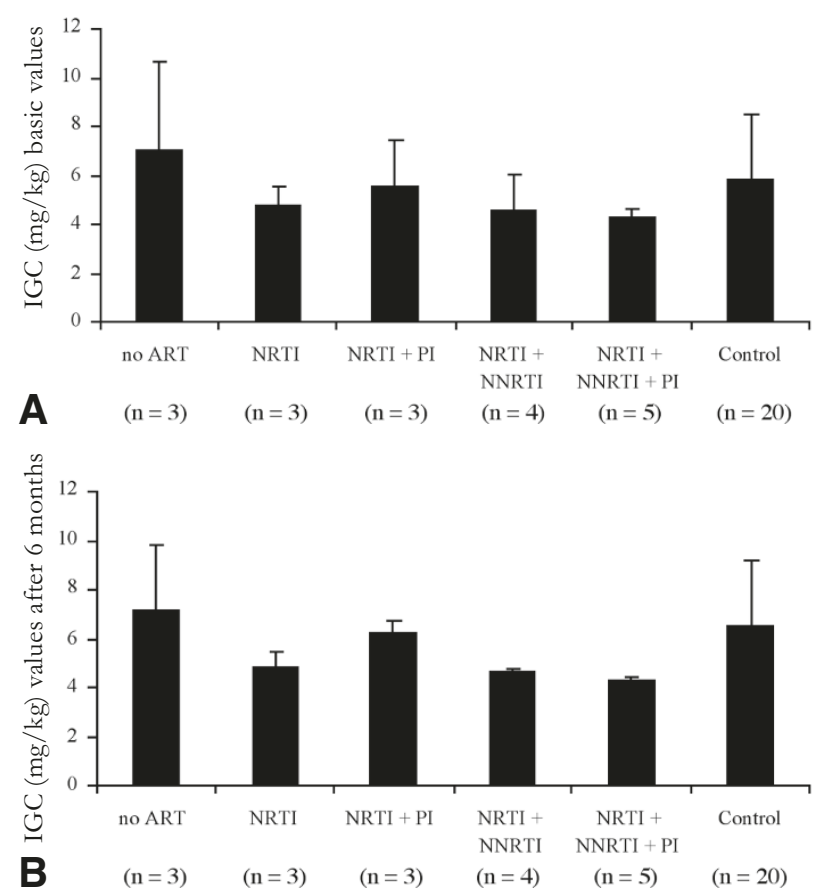

Fig. 2. Indocyanine green clearance - as a parameter of biliary secretion capacity and liver perfusion - was not dependent on the different anti-retroviral regimens in patients with HIV-infection. IGC is expressed as $\mathrm{mg} / \mathrm{kg}: n=$ patients' number. $A$. number. A. mination and course of IGC after 6 months of anti-retroviral treatment. 

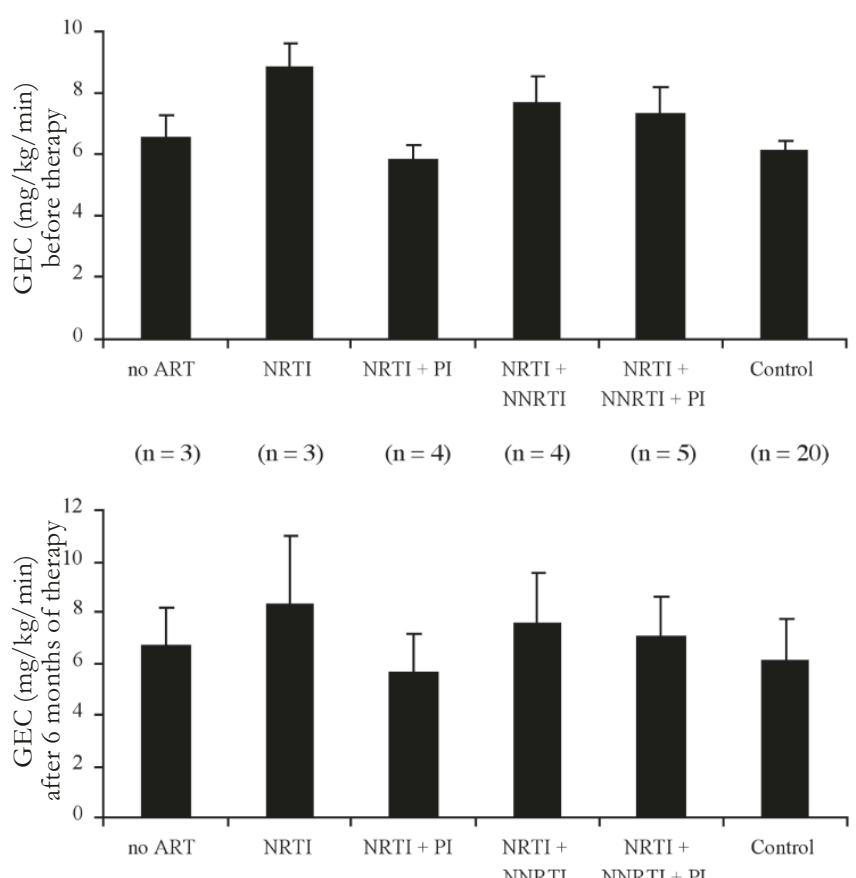

A

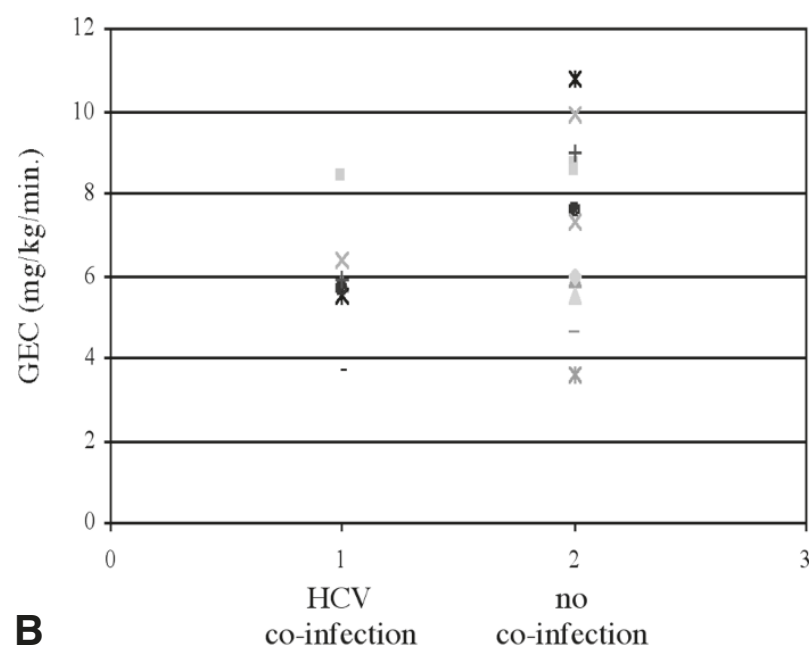

Galactose elimination capacity in HIV-patients was not influenced by ART: To test if metabolic liver function is improved in HIV-patients under ART, GEC was performed in non-treated patients with $H I V$-infection, and values were then compared to the values of treated patients. Again, as demonstrated before for the indocyanine green clearance, no significant differences were observed between HIV-patients before and after the initiation of anti-retroviral therapy (Fig. $3 A$ ).

Galactose elimination capacity in HIV-patients was unaffected by co-infection with hepatitis $\mathrm{C}$ virus. Fife out of 19 patients with HIV-infection were co-infected with hepatitis $\mathrm{C}$ virus (Fig. 3B). During anti-retroviral therapy, values for GEC changed only marginal from $7.7 \mathrm{mg} / \mathrm{kg} / \mathrm{min}$. in HIV-patients without HCV co-in fection as compared to $5.75 \mathrm{mg} / \mathrm{kg} / \mathrm{min}$. in HIV-patients with hepatitis C co-infection. GEC was apparently reduced in patients with HIV-HCV co-infection indicating a reduction of cytosolic liver function. However, this reduction was statistically not significant.

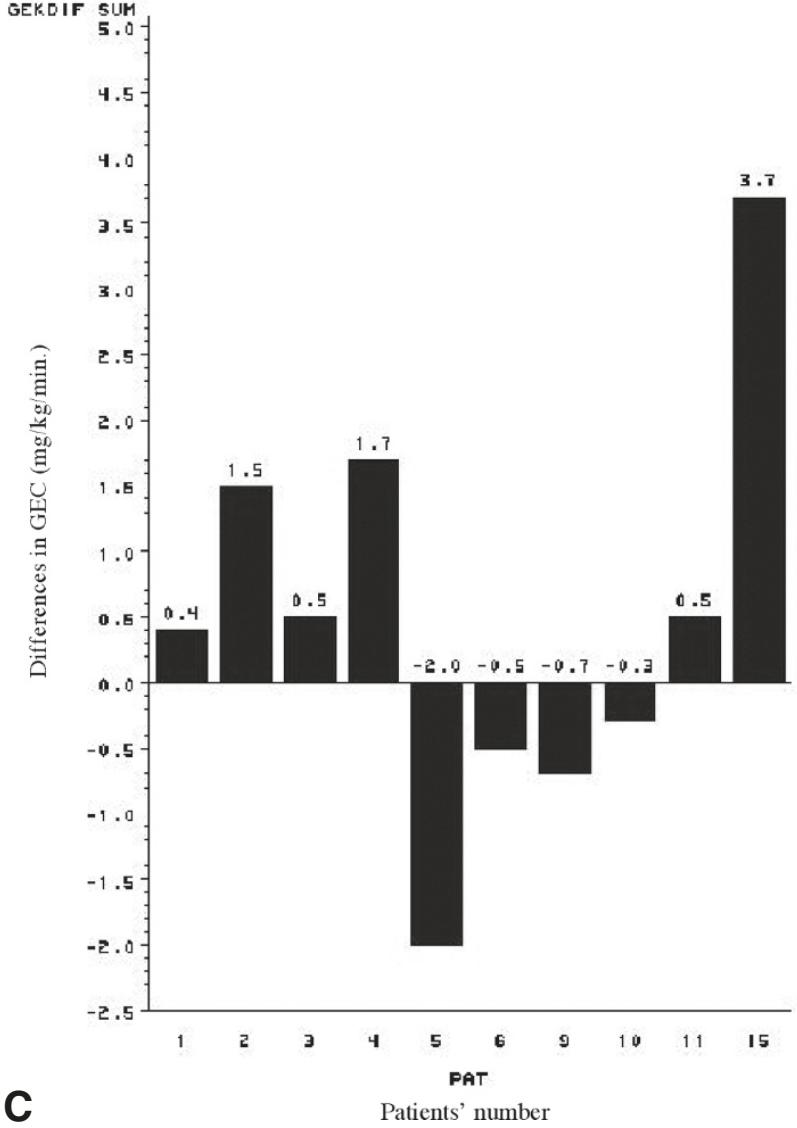

Fig. 3. Measurement of GEC in patients with HIV-infection: A. Galactose elimination capacity - determined before and 6 months after initiation of ART - was not influenced by variou anti-retroviral regimens. B. GEC was not significantly reduced during co-infection with hepatitis $\mathrm{C}$ virus. $\mathrm{C}$. The metabolic liver capacity oscillated under long-term therapy for 3 to 6 months remained unchanged without obvious tendency. $P A T=$ patients' number; GEKDIF = differences in GEC.

Galactose elimination capacity remained unchanged during the course of anti-retroviral treatment: In our final experiment, 10 randomly selected patients with HIV-infection had a follow-up examination after 3 to 6 months of treatment with anti-retroviral therapy. As demonstrated in Figure 3C, the metabolic liver capacity oscillated during this long-term therapy was mainly unchanged without obvious tendency.

\section{DisCUSSION}

Hepatic steatosis is a common feature developing upon application of state-of-the-art anti-retroviral regimens, including nucleoside reverse transcriptase inhibitors (NRTI), non-NRTIs and protease inhibitors. Still, severe hepatotoxicity is a rare event However, with the increasing employment of HAART in developed countries, hepatic steatosis and severe hepatotoxicity with acute liver failure may be seen more often among patients with HIV-infection 
(Montessori, Harris et al. 2003; Kahraman, Miller et al. 2006).

The substantial survival benefit of HAART in the treatment of patients infected with HIV-1 has been frequently documented, revealing both a reduction in morbidity and mortality as well as a significant im provement in the quality of life (Lipsky 1996; Ristig Drechsler et al. 2005). Still, alongside the benefits of these treatments, HAART-associated adverse events become increasingly apparent. These ranged from mild side-effects, such as abdominal pain, polyneuropathy or elevated liver enzymes to severe life-threatening complications, such as acute liver failure, pancreatitis, and lactic acidosis.

Quantitative tests of liver function may allow a prognostic assessment of patients with various live diseases (Herold, Berg et al. 2000; Herold, Heinz et al 2001; Herold, Heinz et al. 2001; Herold, Ganslmaye et al. 2002; Herold, Regn et al. 2002; Herold, Ganslmayer et al. 2003; Jochum, Beste et al. 2006; Reichen 2006). Although transaminases - the commonly used markers of hepatocellular injury - have a high sensitivity in screening for liver diseases, they do not provide an information about prognosis. In addition, assessment of liver function using bilirubin, serum albumin and prothrombin-time is limited by their inability to identify the functional reserve of the liver. Dynamic liver function tests are therefore an improvement on the static tests. They are cost-effective, easy to perform and analyse, safe, have a simple pharmacokinetic profile with minimal drug interactions, have a high predictive value, and $f i$ nally, provide quick results (Jalan and Hayes 1995; Candelli, Cazzato et al. 2004). The value of these tests has been demonstrated for patients with acute reduction of the hepatic metabolic capacity, such as in fulminant liver failure and alcoholic hepatitis (Merkel, Bolognesi et al. 1989). However, the value of QTLF in patients infected with HIV is debatable, since parameters of QTLF could be influenced by different co-factors such as anti-viral drug administration, viral co-infections, and age. Still, there is insufficient data about these patients and a comparison of various QTLF is still lacking. Recent studies have described changes of a single QTLF in patients with chronic hepatitis C-infection receiving interferon therapy (Ocker, Ganslmayer et al. 2005).

In the present study, we determined a panel of three QTLF and, when compared to a healthy control, lidocaine half-life as a parameter of detoxification quality of hepatocytes was found to be significantly reduced in HIV-positive patients without anti-retroviral treatment. A number of medications that are com monly used in HAART regimens are metabolized by the hepatic CYP enzymes, which raises the possibility of significant interactions between anti-retroviral medications and hepatic impairment. The cytochrome $p 450$ isoenzyme CYP1A2 is the main isoform responsible for lidocaine biotransformation. In HIV-positive patients protease inbibitors (for example lopinavir/ ritonavir) can lead to the induction of CYP1A2 which may interact with lidocaine metabolism (Orlando, Piccoli et al. 2004; Wyles and Gerber 2005). We conclude that measuring LHT may be a tool to predict prognosis or com plications in HIV-infected patients with liver disease, and may help to discriminate positive patients' response from negative response. In HIV-positive patients with compromised liver function this might help to modify anti-retroviral therapy and switch to drugs that are less bepatotoxic.

Severe hepatotoxicity in HIV-infected patients receiving $H A A R T$ occurs in $5-10 \%$ of cases. The main risk factors are viral co-infections, advanced liver disease, and elevated liver enzymes at the start of therapy. Anti-retroviral drugs associated with an increased risk of severe hepatotoxicity are stavudine, didanosine, nevirapine, full-dose ritonavir and tipranavir (Vogel and Rockstrob 2005; Vogel and Rockstrob 2007). We observed an increase in the activity of cytochrome $p 450$ in $H I V$-positive patients with different ART regimes which might be a virus- or a drug-induced effect. Combining anti-retroviral therapies shifted cytochrome $p 450$ activity back into standard ranges. However, the different components of HAART are each associated with risks of liver toxicity. Most drugs are metabolized by cytochrome 7450 enzymes in the liver, and this may be affected by liver disease. The mechanisms for drug-induced liver injury include dose-dependent toxicity, idiosyncratic reactions, mitochondrial toxicity, and immune reconstitution. Once diagnosed, management generally involves discontinuation of the offending anti-retroviral drugs. Therefore, QTLF may provide important prognostic informations in patients with HIV-related liver disease and tolerability of HAART (Inductivo-Yu and Bonacini 2008).

Activation of the cytochrome p450 system showed no essential differences in HIV-patients with HCV co-in fection. However, this co-infection seems to be negligible on liver function, and is not a contra-indication for ART. Ocker and colleagues investigated to what extent anti-viral therapy influenced QTLF in patients with $H C V$-infection. Patients with HIV co-infection were unfortunately excluded from the study. Tests were performed before and 3 months after the initiation of ther apy. They demonstrated that parameters of microsomal and cytosolic liver function were reduced in all patients before therapy initiation and returned to normal values in therapy responders after 3 months. Parameters of liver perfusion were not affected by anti-viral therapy. Ac cording to the authors, early determination of GEC may differentiate responders from non-responders to anti-viral treatment in patients with hepatitis C-infection (Ocker, Ganslmayer et al. 2005). In a further study, Herold et al. applied a broad panel of QTLF to patients with hepatitis B and C-infection. Again, patients with HIV co-infection were unfortunately not included in the study. All QTLF values were correlated with liver histology and Child-Pugh classification for liver cirrhosis. The authors could demonstrate that in patients with moderate fibrosis, metabolic liver function was significantly decreased whereas hepatic perfusion remained normal. Severe fibrosis and cirrhosis showed a significant decline in all OTLF. Viral aetiology and HCV genotypes did not change QTLF (Herold, Heinz. et al. 2001).

In our present study, parameters of the cytosolic metabolic capacity, liver perfusion and secretion were not compromised in non-treated versus treated patients with HIV-infection. We examined IGC and 
GEC and found no significant correlation. Therefore, we conclude that viral damage to the liver compromises quality of liver detoxification before metabolic capacity and hepatic perfusion. The great variability of GEC within HIV-infected patients might be related to the small number of patients investigated in this study or to different anti-viral regimens. So far, no data exist about impact of different components of HAART on GEC. Compared to treated patients with HIV-infection, we found a significant reduction in lidocaine halflife before the initiation of anti-retroviral therapy. However, we found no significant influence of bepatitis $C$ co-infection although infection with hepatotropic virus es affects liver function. The reason is obviously the small number of patients investigated with $\mathrm{HIV}-\mathrm{HCV}$ co-infection.

Until now, QTLF have not been validated in patients with HIV-infection. At present, it remains unclear if and how far QTLF may provide importan prognostic informations in patients with HIV-related liver diseases that is superior to that of conventional prognostic parameters or risk scores. Our current study suggests that certain QTLF could be useful to monitor the effect of anti-retroviral or liver-protective therapy in patients with liver disease caused by HIVinfection and additionally, it may be an economical method to follow up these patients - particularly those under treatment with ART. Repeated measurements of lidocaine half-life with a simple device can predict the outcome and therefore, might be clinically useful in following patients with HIV-infection. For example, assessment of IGC removal has been demonstrated in many different clinical settings to have major predictive power with respect to morbidity and mortality (Reichen 1995). It is well known that all anti-retroviral drug classes, nucleoside/nucleotide reverse transcriptase inbibitors (NRTI), non-nucleoside reverse transcriptase inhibitors (NNRTI), and protease inbibitors (PI) mas cause bepatotoxicity but in different pathways (Abrescia, D'Abbraccio et al. 2005). However, in our experiments we did not observe significant changes in quantitative liver tests in dependence to diverse anti-retroviral regimens. So far, we evaluated only a small group of $H I V$-patients. Since QTLF could be useful to monitor anti-retroviral therapy in patients with HIV-infection and regarding the increasing role of liver-related morbidity and mortality in HIV-infected patients, more prospective randomized, controlled trials with a larger number of patients are justified.

Acknowledgement: We would like to thank the anonymous reviewers for their most valuable and insightful suggestions.

\section{REFERENCES}

Abrescia, N., M. D'Abbraccio, et al. (2005). "Hepatotoxicity of antiretroviral drugs." Curr Pharm Des 11(28): 3697-710.

Armuzzi, A., M. Candelli, et al. (2002). "Review article: breath testing for human liver function assessment." Aliment Pharmacol Ther 16(12): 1977-96.

Bradley, S. E., F. J. Ingelfinger, et al. (1945). "The Estimation of Hepatic Blood Flow in Man." J Clin Invest 24(6): 890-7.

Candelli, M., I. A. Cazzato, et al. (2004). "13C-breath tests in the study of mitochondrial liver function." Eur Rev Med Pharmacol Sci 8(1): 23-31.
Clark, J. M., F. L. Brancati, et al. (2002). "Nonalcoholic fatty liver disease." Gastroenterology 122(6): 1649-57.

Ena, J., C. Amador, et al. (2003). "Risk and determinants of developing severe liver toxicity during therapy with nevirapine-and efavirenz-containing regimens in HIV-infected patients." Int J STD AIDS 14(11): 776-81.

Farrell, G. C. (2002). "Drugs and steatohepatitis." Semin Liver Dis 22(2): 185-94.

Herold, C., P. Berg, et al. (2000). "Parameters of microsoma and cytosolic liver function but not of liver perfusion predict portal vein velocity in noncirrhotic patients with chronic hepatitis C." Dig Dis Sci 45(11): 2233-7.

Herold, C., M. Ganslmayer, et al. (2002). "Quantitative testing of liver function compared to prognostic scores in patients with primary biliary cirrhosis." Liver 22(2): 159-65.

Herold, C., M. Ganslmayer, et al. (2003). "Inducibility of microsomal liver function may differentiate cirrhotic patients with maintained compared with severely compromised liver reserve." J Gastroenterol Hepatol 18(4): 445-9.

Herold, C., R. Heinz, et al. (2001). "Quantitative testing of liver function in relation to fibrosis in patients with chronic hepatitis B and C." Liver 21(4): 260-5.

Herold, C., R. Heinz, et al. (2001). "Quantitative testing of liver function in patients with cirrhosis due to chronic hepatitis C to assess disease severity." Liver 21(1): 26-30.

Herold, C., S. Regn, et al. (2002). "Can quantitative tests of liver function discriminate between different etiologies of liver cirrhosis?" Dig Dis Sci 47(12): 2669-73.

Inductivo-Yu, I. and M. Bonacini (2008). "Highly active antiretroviral therapy-induced liver injury." Curr Drug Sa $3(1): 4-13$.

Jalan, R. and P. C. Hayes (1995). "Review article: quantitative tests of liver function." Aliment Pharmacol Ther 9(3): 263-70.

Jochum, C., M. Beste, et al. (2006). "Quantitative liver function tests in donors and recipients of living donor live transplantation." Liver Transpl 12(4): 544-9.

Kahraman, A., M. Miller, et al. (2006). "Non-alcoholic fatty liver disease in HIV-positive patients predisposes for acute-on-chronic liver failure: two cases." Eur J Gastroenterol Hepatol 18(1): 101-5.

Keiding, S. (1987). "Hepatic clearance and liver blood flow." J Hepatol 4(3): 393-8

Lamesch, P., B. Ringe, et al. (1990). "Assessment of live function in the early postoperative period after live transplantation with ICG, MEGX, and GAL tests." Transplant Proc 22(4): 1539-41.

Lebovics, E., S. N. Thung, et al. (1985). "The liver in the acquired immunodeficiency syndrome: a clinical and histologic study." Hepatology 5(2): 293-8.

Lee, G. A., T. Seneviratne, et al. (2004). "The metabolic effects of lopinavir/ritonavir in HIV-negative men." Aids 18: 641-649

Leevy, C. M., C. L. Mendenhall, et al. (1962). "Estimation of hepatic blood flow with indocyanine green." J Clin Invest 41: 1169-79

Lipsky, J. J. (1996). "Antiretroviral drugs for AIDS." Lancet 348(9030): 800-3.

Meijer, D. K., B. Weert, et al. (1988). "Pharmacokinetics of biliary excretion in man. VI. Indocyanine green." Eur Clin Pharmacol 35(3): 295-303.

Merkel, C., M. Bolognesi, et al. (1989). "Indocyanine green intrinsic hepatic clearance as a prognostic index of survival in patients with cirrhosis." J Hepatol 9(1): 16-22

Montessori, V., M. Harris, et al. (2003). "Hepatotoxicity of nucleoside reverse transcriptase inhibitors." Semin Liver Dis 23(2): 167-72

Ocker, M., M. Ganslmayer, et al. (2005). "Improvement of quantitative testing of liver function in patients with chronic hepatitis $C$ after installment of antiviral therapy." World J Gastroenterol 11(35): 5521-4. 
Oellerich, M. and V. W. Armstrong (2001). "The MEGX test: a tool for the real-time assessment of hepatic function." Ther Drug Monit 23(2): 81-92

Orlando, R., P. Piccoli, et al. (2004). "Cytochrome P450 1A2 is a major determinant of lidocaine metabolism in vivo: effects of liver function." Clin Pharmacol Ther 75(1): $80-8$.

Ott, P., O. Clemmesen, et al. (2000). "Interpretation of simultaneous measurements of hepatic extraction fractions of indocyanine green

Plevris, J. N., R. Jalan, et al. (1999). "Indocyanine green cleatance reflects reperfusion injury following liver transplantation and is an early predictor of graft function." J Hepatol 30(1): 142-8.

Reichel, C., G. Wienkoop, et al. (1995). "[Monoethylglycinexylidide (MEGX)-test. A test for the assessment of prognosis before and after liver transplantation]." Dtsch Med Wochenschr 120(6): 179-83.

Reichel, C., G. Wienkoop, et al. (1995). "Which lidocain dose should be used for the MEGX liver function test?" Hepatol 22(5): 600

Reichen, J. (1995). "Assessment of hepatic function with xenobiotics." Semin Liver Dis 15(3): 189-201.

Reichen, J. (2006). "Quantitative liver tests in living live donors and their recipients." Liver Transpl 12(4): 514-5.

Reid, A. E. (2001). "Nonalcoholic steatohepatitis." Gastroenterology 121(3): 710-23

Ristig, M., H. Drechsler, et al. (2005). "Hepatic steatosis an HIV infection." AIDS Patient Care STDS 19(6): 356-65.

Schneider, A. R., W. F. Caspary, et al. (2004). "[(13)C-breath tests for the assessment of liver function]." Z Gastroenterol 42(3): 269-75

Schneiderman, D. J., D. M. Arenson, et al. (1987). "Hepatic disease in patients with the acquired immune deficiency syndrome (AIDS)." Hepatology 7(5): 925-30.
Tygstrup, N. (1963). "Determination of the hepatic galactose elimination capacity after a single intravenous injection in man: the reproducibility and the influence of uneven distribution." Acta Physiol Scand 58: 162-72.

Tygstrup, N. (1966). "Determination of the hepatic elimination capacity (Lm) of galactose by single injection." Scand J Clin Lab Invest Suppl 18: 118-25.

Tygstrup, N., P. K. Andersen, et al. (1985). "Prognostic evaluation in alcoholic cirrhosis." Acta Med Scand Suppl 703: $149-56$

Vogel, M. and J. K. Rockstroh (2005). "[Therapy of hepatitis $B$ and hepatitis C coinfection in HIV patients]." MMW Fortschr Med 147 Spec No 1: 56-9.

Vogel, M. and J. K. Rockstroh (2007). "Hepatotoxicity and liver disease in the context of HIV therapy." Curr Opin HIV AIDS 2(4): 306-13.

Wyles, D. L. and J. G. Gerber (2005). "Antiretroviral drug pharmacokinetics in hepatitis with hepatic dysfunction." Clin Infect Dis 40(1): 174-81.

Yano, E., K. Tagawa, et al. (2001). "Test validity of periodic liver function tests in a population of Japanese male bank employees." J Clin Epidemiol 54(9): 945-51.

Received: October 15, 2008 / Accepted: May 27, 2009

Address correspondence:

Gerken Guido, MD, Professor of Medicine

Department of Gastroenterology and Hepatology

University Hospital Essen

Hufelandstr. 55

45122 Essen

Germany

Phone: + 49 (0)201/723-3610

Fax: $\quad+49(0) 201 / 723-5971$

E-mail: guido.gerken@uk-essen.de 